\title{
Inventions of scientists, engineers and specialists from different countries in the area of nanotechnologies. Part V ${ }^{1)}$
}

\author{
Leonid A. Ivanov ${ }^{1 *}$ (D), Li D. Xu² (D), Konstantin E. Razumeev³ (iD, Valentina M. Feoktistova ${ }^{4}$ (iD, Petr S. Prokopiev ${ }^{5}$ \\ ${ }^{1}$ Russian Academy of Engineering, Moscow, Russia \\ ${ }^{2}$ Old Dominion University, Norfolk, Virginia, USA \\ ${ }^{3}$ A.N. Kosygin Russian State University (Technology. Design. Art), Moscow, Russia \\ ${ }^{4}$ Russian State University of Tourism and Service; Cherkizovo, Moscow region, Russia \\ ${ }^{5}$ Financial University Under the Government of the Russian Federation, Moscow, Russia \\ *Corresponding author: e-mail: L.a.ivanov@mail.ru
}

\begin{abstract}
Introduction. Advanced technologies impress people's imagination demonstrating the latest achievements (materials, methods, systems, technologies, devices etc.) that dramatically change the world. This, first of all, concerns nanotechnological inventions designed by scientists, engineers and specialists from different countries. Main part. The article provides an abstract overview of inventions of scientists, engineers and specialists from different countries: Russia, USA, China, Belarus, Italy. The results of the creative activity of scientists, engineers and specialists, including inventions in the field of nanotechnology and nanomaterials allow, when introduced to industry, achieving a significant effect in construction, housing and communal services, and related sectors of the economy. For example, the invention «Protective coating and method of its application» relates to the field of protecting metals from corrosion. To obtain a protective coating, a composition containing an industrial oil with a powder of a phosphorusmodified copper-carbon nanocomposite is applied to the surface of an unalloyed steel and subjected to thermochemical activation by heating for 20 minutes at a temperature of $100-200^{\circ} \mathrm{C}$. During the formation of the coating, strong donor-acceptor bonds of iron atoms with phosphorus, which are in the composition of the copper-carbon nanocomposite, are formed. In the course of experiments, it was found that heating samples with a coating of oil with the addition of a phosphorus-modified copper-carbon nanocomposite to $100-200^{\circ} \mathrm{C}$ increases the chemical activity of the nanocomposite, as a result of which a protective coating forms on the surface, which reduces the corrosion rate depending on the concentration of the modified metal-carbon nanocomposite. by $70-95 \%$. The following inventions in the field of nanotechnology are also of interest to specialists: a method for obtaining a nanostructured surface of a metal workpiece by laser treatment, a device for producing nanodispersed metal oxides, a method for producing a nanostructured composite material based on aluminum, a method for modifying the surfaces of plates of a brazed plate heat exchanger, etc. Conclusion. One of the most challenging tasks the economy of every country face is to increase industrial competitiveness through technological upgrade. From the side of the state and companies the principal object to control in this process are the people and enterprises dealing with introduction of inventions and new technologies.
\end{abstract}

KEYWORDS: nanotechnologies in construction, nanoscale materials, nanocomposites, nanodispersed metal oxides, nanostructuring. FOR CITATION: Ivanov L.A., Xu L.D., Razumeev K.E., Feoktistova V.M., Prokopiev P.S. Inventions of scientists, engineers and specialists from different countries in the area of nanotechnologies. Part V. Nanotechnologies in Construction. 2021; 13(5): 311-318. https://doi. org/10.15828/2075-8545-2021-13-5-311-318

\section{INTRODUCTION}

A dvanced technologies impress people's imagination demonstrating the latest achievements (materials, methods, systems, technologies, devices etc.) that dramatically change the world. This primarily concerns nanotechnological inventions designed by scientists, engineers and specialists from different countries.

\footnotetext{
1) Continuation. Part I - Part IV have been published in the journal «Nanotechnologies in Construction», volume 13, issues 1-4, 2021. (c) Ivanov L.A., Xu L.D., Razumeev K.E., Feoktistova V.M., Prokopiev P.S., 2021
} 


\section{MAIN PART}

\section{A method of obtaining a nanostructured surface of a metal workpiece by laser processing (RU 2752821 C1)}

Nanoscale structures are characterized by the peculiarities that the processes under consideration and the actions performed take place in the nanometer range of spatial dimensions, where the initial material is individual atoms, molecules, and molecular systems. Therefore, in contrast to traditional technology, nanotechnology is characterized by an "individual" approach, in which external control reaches individual atoms and molecules, which makes it possible to create nanoscale materials from them with a controlled structure and fundamentally new physicochemical properties - optical, electrical, magnetic, corrosion-resistant, including those that improve the mechanical and tribotechnical properties of the surface. Based on the foregoing, the new achieved technical result of the proposed invention is provided by the following technical advantages over the prototype [1]:

1. Improved quality of joining of dissimilar (different in chemical composition) metal workpieces during diffusion welding is achieved - an increase by at least $14 \%$ of the ultimate strength of the welded joint due to preliminary nanostructuring of the surface of the metal workpiece is obtained.

2. During nanostructuring, a change in the initial physicochemical properties of the surface of a metal workpiece is provided - adhesive, optical, electrical, magnetic, corrosion-resistant, including those that improve the mechanical and tribotechnical properties of the surface, due to the formation of micron and submicron structures with a single scanning focused laser beam. The resulting surface structures have the form of micro-furrows, micropores, curved channels, microcones with transverse dimensions from 1 to $5 \mu \mathrm{m}$, as well as spherical structures with a diameter of about $500 \mathrm{~nm}$ on cylindrical supportslegs (microjets) with a width of about $300 \mathrm{~nm}$.

\section{Foam gel (RU 2753652 C1)}

The invention relates to the field of obtaining a foamed silica gel, structured with nanoadditives, which can be used as a low-density stemming of blast holes or boreholes when crushing a rock mass in the extraction of solid minerals [2].

A foam gel comprising aqueous solutions of liquid glass, a coagulator and a foaming agent, mixed with compressed air, and used in the construction of a borehole charge as a dam, an inert gap and a bottom compensator, characterized in that, in order to increase stability, it additionally contains a silicasol nanoadditive "Nanosil-30", as a coagulator contains a mixture of iron chloride and phosphoric acid, and as a foaming agent

The technical result is achieved by the fact that the most accessible and cheapest component sodium silicate $10-15 \%$ of the mass is used as the starting materials; as a nanoadditive is used "Nanosil-30" $0.05-0.15 \%$ of the mass, which enhances the sol-gel process; orthophosphoric acid $5-8 \%$ of the mass; ferric chloride $0.1-0.3 \%$ of the mass; foaming agent ABSK $3-5 \%$ of the mass and water $71.55-81.85 \%$ of the mass. Depending on the amount of compressed air, the foam gel ratio varies from 5 to 15 units. Since it is known that foam gel of medium and high expansion is not effective for stemming blastholes, that is, it does not ensure the locking of the explosion products.

\section{High performance metal alloy for additive manufacturing of machine parts (RU 2750946 C1)}

Internal combustion engine parts such as gas turbine parts (eg blades, nozzles, shrouds, combustion chambers) must be made of metal alloys designed to withstand high temperature operating conditions. This is especially true for parts that are located close to the combustion chambers of the gas turbine, such as the turbine nozzles. The temperature of the working gas generated by the combustion products in the first stage nozzles can be $1100^{\circ} \mathrm{C}$ or higher, while in the downstream turbine stage the temperature drops to about $650-700^{\circ} \mathrm{C}$.

Special high-temperature nickel-based alloys are used to produce rotating parts such as turbine first stage blades. These alloys are costly, but are required in terms of the need to withstand the combined stress of high temperature and high dynamic stresses generated in the rotor part of the turbomachine.

The invention relates to metallurgy, namely to a metal alloy with high performance characteristics and can be used for the additive production of machine parts, in particular a gas turbine nozzle [3]. A metal alloy for the manufacture of a gas turbine nozzle by the method of additive production, consisting of, wt\%: $\mathrm{O} 0.01-0.05$, $\mathrm{N} 0.005-0.025$, S less than 0.003 , C $0.005-0.07$, Mn 0.60 , 8, Si 0.8-1.0, P less than 0.04, Cr 27-33, Ni 11-12, W 5-9, Fe 0.4-0.7, Ta less than 0.001, B less than 0.003, $\mathrm{Cu}$ less than $0.001, \mathrm{Zr}$ less than 0.003 , Co - the rest. The alloy is characterized by high performance characteristics, namely high resistance to high-temperature oxidation and corrosion, high thermal fatigue resistance, and satisfactory weldability.

\section{Device for producing nanodispersed metal oxides (RU 2752756 C1)}

The invention relates to the chemical industry and metallurgy [4]. The device for obtaining nanodispersed 
metal oxides (Fig. 1) contains a line 1 for the preparation of precursors and a line 2 for hydrothermal synthesis, equipped with reactors, centrifuges and containers. Line 1 includes the first reactor 3 - for preparing the precipitant solution, connected to the second reactor $6-$ for preparing the precursor suspension. Reactor 3 is connected to vessel 4 with a precipitant and vessel 5 with distilled water. A measuring tank 7 with a nitrate salt solution is connected to the reactor 6 . Reactor 6 is connected to decanter 8 , which in turn communicates with tank for decantate 9 and with first centrifuge 10. Line 2 is equipped with reactor 11 for hydrothermal synthesis, connected by means of elevator 12 to the first centrifuge 10 . Tank 5 is connected to the inlet of reactor 11. distilled water, connected to the decanter 8 , and to the outlet of the reactor 11 is connected the second centrifuge 10 , the outlet of which is connected to the dryer 13. Reactors 3 and 6 are made in the form of closed containers, on the outer sides of which are mounted heat exchange jackets, and inside - anchor-type agitators. The reactor 11 is made in the form of an autoclave, installed on a stirring device and connected to the controller with the ability to set and maintain a predetermined temperature regime. The device allows you to obtain various nanodispersed metal oxides

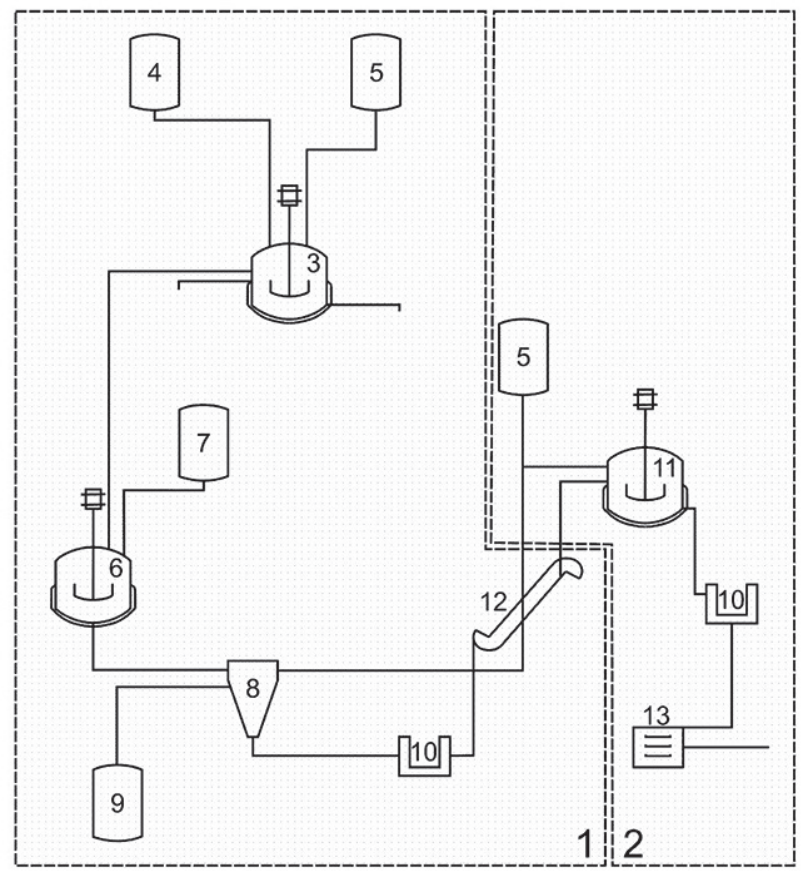

Fig. 1. Technological scheme for obtaining nanodispersed metal oxides by the method of hydrothermal synthesis, where 1 - precursor preparation line, 2 - hydrothermal synthesis line, 3 - first reactor, 4 - vessel with precipitant, 5 - vessels with distilled water, 6 - second reactor, 7 - measuring tank , 8 - decanter, 9 - container for decantate, 10 - centrifuges, 11 - third reactor, 12 - elevator, 13 - dryer. without re-equipment, maintain optimal technological modes and reliably control the process.

The technical result is achieved due to the fact that tanks with a precipitant and with distilled water are connected to the first reactor, a measuring tank with a solution of nitrate salt is connected to the second reactor, while this reactor is connected to a decanter, which, in turn, is connected to a tank for decantate and with the first centrifuge, and the device additionally contains a hydrothermal synthesis line equipped with a third reactor - for hydrothermal synthesis, connected by means of a lift with the outlet of the specified centrifuge, a container with distilled water connected to a decanter is connected to the inlet of the third reactor, and a second centrifuge is connected to the outlet of the third reactor, the outlet of which is connected to the dryer. In particular, the first and second reactors are designed as closed containers. In particular, heat exchange jackets are mounted on the outside of the first and second reactors. In particular, anchor-type agitators are mounted in the first and second reactors. In particular, the third reactor is designed as an autoclave. In particular, the third reactor is mounted on a mixing device. In particular, the third reactor is connected to the controller with the ability to set and maintain a predetermined temperature regime.

\section{Method of welding parts made of heat-resistant nickel-based alloys using laser radiation (RU 2752822 C1)}

In power plants, two-layer welded pipes made of heatresistant nickel alloy and heat-resistant bronze with high thermal conductivity are often used, and they are joined together by diffusion welding. Although the implementation of diffusion welding in a vacuum or in an inert gas atmosphere expands the technological capabilities of this welding method, at present the problem of improving the quality of the joint and expanding the permissible temperature regime of welding is still urgent.

Improvement of the mechanical characteristics of welded joints during diffusion welding is possible due to the formation of various ordered structures on the surfaces to be welded, including the micron and submicron scale. To create them, such technological methods as laser modification of the surfaces of the workpieces being welded can be used. Direct laser nanostructuring with nanosecond pulses can become a promising method for the formation of a nanostructure on a metal surface. The ultraviolet region of the spectrum of laser radiation is more preferable due to the higher absorption of radiation by metals compared to the visible and infrared regions of the spectrum. The effective effect of laser radiation on a thin $(\sim 1 \mu \mathrm{m})$ surface layer of a metal makes it possible to obtain various micro- and nanostructures with specified parameters. 
The invention relates to a method for welding parts made of heat-resistant alloys on a nickel base and can be used in various sectors of mechanical engineering and metallurgy [5]. The technical result of the invention is to improve the quality of the connection of the parts to be welded. The method includes processing with laser radiation of a nanosecond pulsed laser at a cooling rate of the nanostructured surface, providing the formation of relief structures on it with a size of less than $100 \mathrm{~nm}$. The overlap ratio of the laser beam spots is defined as the ratio of the area treated with two laser pulses to the area of one laser beam spot. After nanostructuring the surfaces of parts made of high-temperature nickel-based alloys, their diffusion welding is performed in a sealed chamber by the action of pressure and heating of the parts to be welded from high-temperature nickel-based alloys in a vacuum or in an inert gas environment.

\section{A method for producing a nanostructured composite material based on aluminum (RU 2751401 C2)}

The invention relates to a method for producing a nanostructured composite material based on aluminum modified with C60 fullerene, with improved physical and mechanical properties, for use in mechanical engineering and aerospace [6].

Aluminum-based nanostructured composite materials modified with $\mathrm{C} 60$ fullerene demonstrate an increased level of mechanical properties. For nanostructured composites of this kind, it is known that with a decrease in the average crystallite size of the matrix, the strength properties increase, while at the initial stages there is a laminar decrease in plasticity proportional to the decrease in the average crystallite size, and then, when the critical value of the average crystallite size is reached, there is a sharp decrease in plasticity almost to zero values. Therefore, the control of the properties of bulk nanostructured composite materials only by reducing the structural elements of the material is severely limited.

The problem to be solved by this invention is aimed at creating a nanostructured composite material with a duplex structure based on aluminum modified with C60 fullerene, with improved physical and mechanical properties for use in mechanical engineering and the aerospace industry. The task is solved due to the precise control of the chemical and phase composition of the powders, ensuring the average size of aluminum crystallites $\leqslant 70 \mathrm{~nm}$ in a fine-crystalline powder and in the range of $70-150 \mathrm{~nm}$ in a coarse-crystalline one, due to strict adherence to the 1: 1 ratio of two types of powders in the final material, as well as due to their mixing at the level of individual crystallites. Powder of fullerene C60 in crystalline form in an amount of $0.1-5-0.5 \mathrm{wt} . \%$ Is used as a modifying carbon phase.
The technical result is to increase the plasticity of a nanostructured composite material with a duplex structure based on aluminum modified with $\mathrm{C} 60$ fullerene, with insignificant losses in strength characteristics. A method for producing a nanostructured composite material with a duplex structure based on aluminum includes several stages of component preparation: 1 - preparation of coarse-crystalline powders; 2 - preparation of fine crystalline powders; 3 - mixing coarse and fine crystalline powders; 4 - consolidation of the obtained powder mixture by the method of severe plastic deformation direct hot extrusion.

\section{A method for producing a composite material with oriented carbon nanotubes (RU 2751882 C1)}

The invention relates to the field of production of composite materials, consisting of a reinforcing material, a polymer matrix and a filler, in the role of which are carbon nanotubes, and can be used to create composite materials with increased strength due to the use of a uniform constant electric field that destroys agglomerates of carbon nanotubes and orienting nanotubes [7]. A method for producing a composite material with oriented carbon nanotubes, which consists in creating a polymer composite material with oriented nanotubes using an electric field, where the reinforcing material is passed through an impregnating bath containing a polymer binder and carbon nanotubes in the direction of the intensity vector of a uniform constant electric field that occurs when a constant electric field is applied. electric current, destroying agglomerates of carbon nanotubes and orienting the nanotubes along the direction of movement of the reinforcing material.

The technical result is achieved by the fact that in the method of producing a composite material with oriented carbon nanotubes, which consists in creating a polymer composite material with oriented nanotubes using an electric field, the reinforcing material is passed through an impregnating bath containing a polymer binder and carbon nanotubes in the direction of the uniform constant electric stress vector. field arising when a direct electric current is applied, destroying agglomerates of carbon nanotubes and orienting the nanotubes along the direction of movement of the reinforcing material.

The invention is illustrated in Fig. 2 and Fig. 3.

The method of producing a composite material with oriented carbon nanotubes consists in creating a polymer composite material with oriented nanotubes using an electric field, while the reinforcing material 1 is passed through an impregnating bath 2 containing a polymer binder 11 and carbon nanotubes 10 in the direction of the intensity vector of a uniform constant electric field, arising from the supply of direct electric current, destroying the agglomerates of carbon nanotubes 10 and orienting 


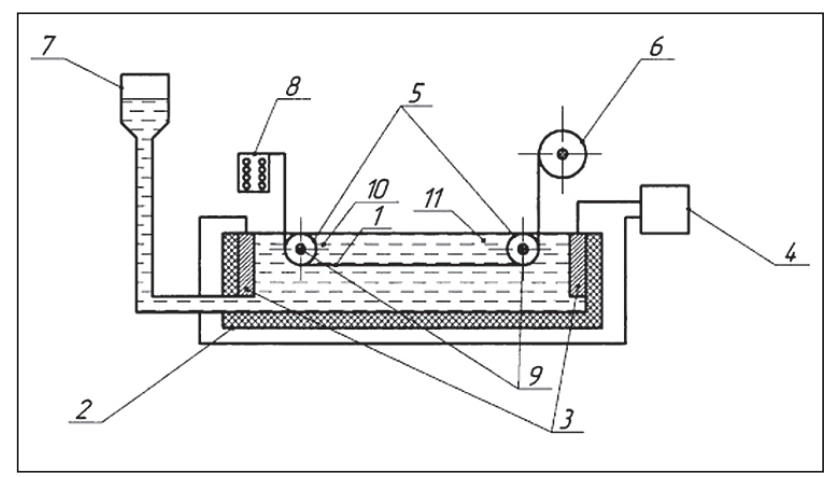

Fig. 2. Schematic diagram of the installation, where 1 - reinforcing material, 2 - impregnating bath, 3 - covers, 4 - orienting block, 5 - rollers, 6 - mandrel, 7 - container with a binder, 8 - creel, 9 - axes, 10 - carbon nanotubes, 11 - polymer binder

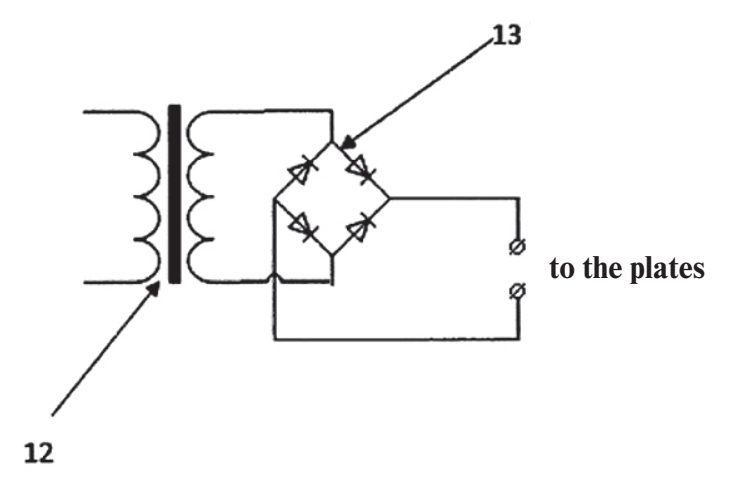

Fig. 3. Schematic diagram of the orienting unit, where 12 - transformer, 13 - diode bridge

the nanotubes along the direction of movement of the reinforcing material 1 .

\section{Protective coating and method of its application (RU 2752488 C1)}

The invention relates to the field of protection of metals from corrosion [8]. The protective coating of the surface of unalloyed steel contains 5-75 wt.\% Of the powder of a phosphorus-modified copper-carbon nanocomposite, distributed in industrial oil I-20. To obtain a protective coating, a composition containing industrial oil I-20 with 5-75 wt.\% Of powder modified with phosphorus coppercarbon nanocomposite is applied to the surface of unalloyed steel, and subjected to thermochemical activation by heating for 20 minutes at a temperature of $100-200^{\circ} \mathrm{C}$. During the formation of the coating, strong donor-acceptor bonds of iron atoms with phosphorus, which are in the composition of the copper-carbon nanocomposite, are formed, which, according to the X-ray electron spectros- copy spectra, are stable at room temperature and when heated to $500^{\circ} \mathrm{C}$ and above. The invention provides a high degree of protection of the surface of unalloyed steel from corrosion by obtaining strong chemical bonds between the atoms of the anticorrosive coating and its strong chemical adhesion to the surface of the steel.

The objective of the claimed invention is to simplify the composition of the resulting coating as much as possible in terms of the number of components and to use the high chemical and adsorption activity of phosphorusmodified metal-carbon nanocomposites. To achieve this task, we used a phosphorus-modified metal-carbon nanocomposite obtained by the method of mechanochemical interaction of copper-carbon nanostructures with ammonium polyphosphate. Copper-carbon nanocomposite is a nanosized copper-containing cluster stabilized by carbon fibers consisting of polyacetylene and carbine fragments with unpaired electrons at the fragments' joints. Due to this structure, the initial nanocomposite easily reacts with ammonium polyphosphate, as a result of which phosphorus is reduced and enters the carbon shell of the copper-carbon nanocomposite between the fragments of carbon fibers. In this case, phosphorus can be a linking unit (linker) with substances on the metal surface.

The technical result is achieved by the fact that the powder of this modified copper-carbon nanocomposite was mixed with the base mineral oil I-20, which in its original state (in its pure form) does not have noticeable protective properties.

By mixing the I-20 oil with the powder of a phosphorus-modified copper-carbon nanocomposite, oil compositions containing from 5 to $75 \%$ of the nanocomposite were obtained. Preliminary experiments have shown that the introduction of a phosphorus-modified copper-carbon nanocomposite into mineral oil, although it increases the effectiveness of the protective coating, but it helps to reduce the corrosion rate of steel by $10-30 \%$. In the course of experiments, it was found that heating samples with a coating of oil with the addition of a phosphorusmodified copper-carbon nanocomposite to $100-200^{\circ} \mathrm{C}$ increases the chemical activity of the nanocomposite, as a result of which a protective coating forms on the surface, which reduces the corrosion rate depending on the concentration of the modified metal-carbon nanocomposite. by $70-95 \%$.

\section{Semiconductor photoelectric converter (RU 2750366 C1)}

Solar energy is one of the fastest growing industries in the world economy, with an average annual growth rate of $20 \%$. And since the cost of electricity generated by photovoltaic modules is still quite high (installed power $\sim \$ 1 / \mathrm{W}$, electricity cost $\$ 0.071 / \mathrm{W}$ ), ways to make it cheaper by increasing the efficiency of converting solar 
radiation into electricity and increasing the generated electricity are very urgent tasks.

The invention relates to the field of direct conversion of light into electrical energy [9]. A solar cell is a multilayer structure for converting light and a layer with nanoparticles deposited on, or inside, or under an antireflection coating layer, which have photoluminescence properties under the influence of UV radiation. Since photoluminescence occurs in the region of visible light with a lower quantum energy, the flow of heat into the solar cell is reduced. To maintain or improve the efficiency of light conversion, nanoparticles must have a quantum efficiency of photoluminescence $K \geqslant 1$, where $K$ is determined by the proposed calculation formula, which relates the absorption edge of the UV range by nanoparticles; the wavelength of the photoluminescence spectrum at the half-width of the photoluminescence peak, and the spectral response of the semiconductor photoelectric converter at the light wavelength $\lambda$. The design of the solar cell according to the invention increases the power generation of the solar cell by reducing the operating temperature of the cell.

The technical result of the invention is to increase the efficiency of conversion of light energy into electricity under operating conditions when the temperature of the solar cell rises and the conversion efficiency falls. The technical result is achieved due to the fact that in a semiconductor photoelectric converter containing a barrier layer for separating charge carriers in the form of a pn junction or heterojunction, an antireflection coating, a layer of nanoparticles and metallized contacts, where nanoparticles deposited on, or inside, or under a layer of an antireflection coating, have the ability to photoluminescence under the influence of UV light with a wavelength in the range of optimal sensitivity of the photoelectric converter and the quantum efficiency of photoluminescence $\mathrm{K}$ not less than 1 , where $\mathrm{K}$ is determined by the proposed calculation formula.

\section{Method for modifying the surfaces of plates of a brazed plate heat exchanger (RU 2754338 C1)}

The invention relates to nanotechnology and nanostructures, in particular, to a method for producing a modified layer containing nanosized diamond phases and can be used for mechanical engineering and power engineering [10]. The objective of the invention: optimization of the parameters of the technical process for improved modification of selective areas of the surfaces of the BPHE plates by using stencils in a vacuum chamber before further joining the plates to each other by soldering or sintering.

The technical result is achieved by hardening the surface and reducing hydrodynamic losses when the content of diamond phases in the modified layer is from 5 to $15 \%$, which leads to an increase in the service life, a decrease in the load on the hydraulic system and an increase in the efficiency of heat transfer of the BPHE. The combination of physical features of the interaction of laser radiation with graphite makes it possible to form a modified layer containing carbon and diamond phases. The presence of diamond phases in the composition of the modified layer determines its high wear resistance and a decrease in hydrodynamic resistance.

A method for modifying the surfaces of plates of a brazed plate heat exchanger includes placing a plate and a carbon-containing target in a vacuum chamber, pumping out the chamber to the required vacuum level, laser processing the target, followed by condensation of a modified layer containing diamond phases on the surface of the plate. In this case, the application of the modified layer on the surface of the heat exchange plates is carried out by evaporation in a vacuum of $10-5$... 10-7 $\mathrm{mmHg}$ of a carbon-containing target with pulsed laser radiation, for processing the target, laser radiation with a wavelength of 190 to $310 \mathrm{~nm}$, a pulse duration of 1 to $100 \mathrm{~ns}$ with a repetition rate from 10 to $100 \mathrm{~Hz}$ and an energy density on the target surface from 1 to $10 \mathrm{~J} / \mathrm{cm}^{2}$. The content of diamond phases in the modified layer is from 5 to $15 \%$, and the thickness of the modified layer is from 30 to $300 \mathrm{~nm}$.

The implementation of the invention is shown in Fig. 4. A BPHE plate is installed in the vacuum chamber 1 . The laser beam 7 enters the chamber through the vacuum window 6 and is focused on the target surface 9 . The target material evaporated as a result of laser action is transferred in the form of an expanding plasma cloud 8 to the BPHE plate surface and condenses in the form of a modified layer 3. Areas intended for applying solder paste 4 are protected from applying a modified layer using a stencil 5. A stencil is placed above the surface to be treated, the task of which is to protect the areas of the

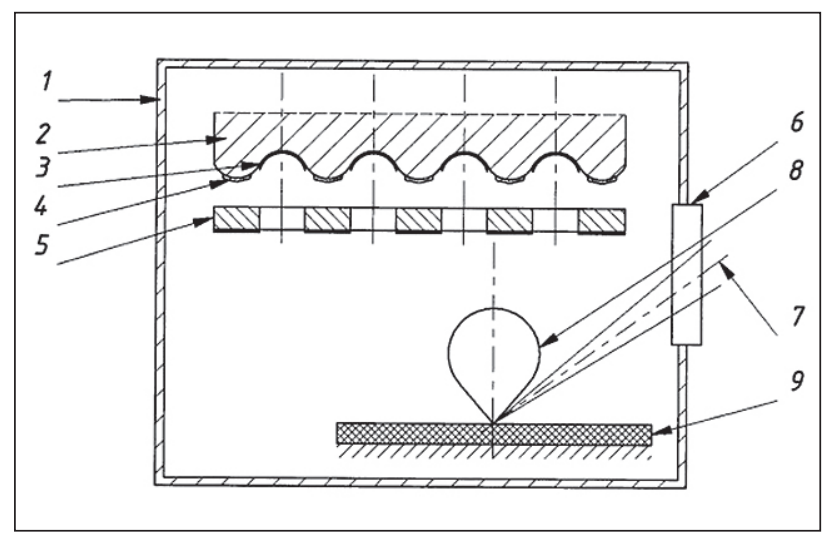

Fig. 4. Schemes for applying a modified layer, where 1 - vacuum chamber, 2 - BPHE plate, 3 - modified layer (ML), 4 - solder paste, 5 - stencil, 6 - vacuum window, 7 - laser radiation, 8 - plasma cloud, 9 - target 
plate from applying MS to areas intended for subsequent formation of a junction. Thus, the MS will be applied to the surface areas of the PPTO plate that are in contact with the liquid during operation.

The specialists can also be interested in the following inventions related to nanotechnologies:

- A method to modify concrete with complex additive which includes hydrothermal nanoparticles $\mathrm{SiO}_{2}$ and multilayer carbon nanotubes [11].

- Method of obtaining gold nanoparticles [12].

- Vacuum gas oil hydrotreating method [13].

- Method of obtaining nanocrystalline epsilon phase of iron oxide [14].

- A method to produce strengthened nanocomposite with additional properties [15].

- Growing nanotubes from free atoms [16].

- Nanoscale pulse generator [17].

- An independent free-standing graphene film and a method for its preparation [18].

- Nano-engineering of construction materials using molecular dynamics simulations [19].
- A method for transferring whisker nanocrystals to a substrate [20].

- A method of obtaining an anticorrosive coating on articles made of monolithic titanium nickelide [21].

- Integral optical sensor for the determination of impurities in gas-air environments [22].

- Development of Nanotechnology in the World and Nanotechnology Standards in Turkey [23].

- Raw material mixture for the manufacture of largesized billets of superhard composite material [24].

\section{CONCLUSION}

One of the most challenging tasks the economy of every country face is to increase industrial competitiveness through technological upgrade. From the side of the state and companies the principal object to control in this process are the people and enterprises dealing with introduction of inventions and new technologies. Therefore, we hope that the information published in this section will be in demand and useful for specialists.

To be concluded

\section{REFERENCES}

1. Yolkin V.N., Malinsky T.V., Mikolutsky S.I. et al. Method of obtaining a nanostructured surface of a metal workpiece by laser processing. Patent RF 2752821. 2021-08-06.

2. Katanov I.B., Katanova N.A. Foam gel. Patent RF 2753652. 2021-08-09.

3. Dimatteo Antonella, Giovannetti Jacopo. Metal alloy with high performance characteristics for additive manufacturing of machine parts. Patent RF 2750946. 2021-07-06.

4. Sharikov F.Yu., Ivanov V.K., Sharikov Yu.V. Device for producing nanodispersed metal oxides. Patent RF 2752756. 2021-08-02.

5. Malinsky T.V., Mikolutsky S.I., Rogalin V.E. et al. Method of welding parts made of high-temperature alloys on a nickel base using laser radiation. Patent RF 2752822. 2021-08-06.

6. Evdokimov I.A., Gryaznova M.I., Bagramov R.Kh. et al. A method of obtaining a nanostructured composite material based on aluminum. Patent RF 2751401. 2021-07-13.

7. Krasnovsky A.N., Kishchuk P.S. A method of obtaining a composite material with oriented carbon nanotubes. Patent RF 2751882. 2021-07-19.

8. Reshetnikov S.M., Shabanova I.N., Kodolov V.I. et al. Protective coating and method of its application. Patent RF 2752488. 2021-07-28.

9. Eidelman B.L., Eidelman K.B., Gudkov D.V., Polisan A.A. Semiconductor photoelectric converter. Patent RF 2750366. 2021-06-28.

10. Shupenev A.E., Korshunov I.S., Fokin Yu.O., Onishchenko D.O. Method for modifying the surfaces of plates of a brazed plate heat exchanger. Patent RF 2754338. 2021-09-01.

11. Ivanov L.A., Xu L.D., Pisarenko Zh.V., Nguyen C.T., Muminova S.R. Inventions of scientists, engineers and specialists from different countries in the area of nanotechnologies. Part IV. Nanotechnologies in Construction. 2021; 13(4): 242-251. https://doi.org/10.15828/2075-8545-2021-13-4-242-251.

12. Belotitskiy V.I., Kumzerov Yu.A., Sysoeva A.A. Method of obtaining gold nanoparticles. Patent RF 2754227. 2021-08-30.

13. Loginova A.N., Morozova Ya.V., Bakanev I.A. et al. Method of vacuum gas oil hydrofining. Patent RF 2753597. 2021-08-18. 
14. Shanenkov I.I., Sivkov A.A., Tsimmerman A.I. et al. Method of obtaining nanocrystalline epsilon phase of iron oxide. Patent RF 2752330. 2021-07-06.

15. Ivanov LA., Razumeev K.E., Bokova E.S., Muminova S.R. The inventions in nanotechnologies as practical solutions. Part V. Nanotechnologies in Construction. 2019; 11(6): 719-729. https://doi.org/10.15828/2075-8545-201911-6-719-729.

16. Laubscher Brian. Growing nanotubes from free atoms. Patent RF 2753099. 2021-08-11.

17. Gurovich B.A., Prikhodko K.E., Kuleshova E.A., Kutuzov L.V. Nanoscale pulse generator. Patent RF 2753276. 2021-08-12.

18. Gao Chao, Peng Li. Independent free-standing graphene film and a method for its preparation. Patent RF 2753510. 2021-08-17.

19. Ivanov L.A., Xu L.D., Bokova E.S., Ishkov A.D., Muminova S.R. Nanotechnologies: are view of inventions and utility models. Part V. Nanotechnologies in Construction. 2020; 12(6): 331-338. https://doi.org/10.15828/20758545-2020-12-6-331-338.

20. Sinev I.V., Timoshenko D.A., Simakov V.V., Zakharevich A.M. Method for transferring whisker nanocrystals to a substrate. Patent RF 2754127. 2021-08-27.

21. Marchenko E.S., Baigonakova G.A., Yasenchuk Yu.F. et al. Method of obtaining anticorrosive coating on articles made of monolithic titanium nickelide. Patent RF 2751704. 2021-07-15.

22. Okhlopkov K.I., Shafirin P.A., Shorokhov A.S., Fedyanin A.A. Integral optical sensor for determining impurities in gas-air environments. Patent RF 2751449. 2021-07-14.

23. Ivanov L.A., Xu L.D., Pisarenko Zh.V., Wang Q., Prokopiev P.S. Inventions of scientists, engineers and specialists from different countries in the area of nanotechnologies. Part II. Nanotechnologies in Construction. 2021; 13(2): 79-89. https://doi.org/10.15828/2075-8545-2021-13-2-79-89.

24. Malchukov V.V., Metsker E.A., Andrianov M.A. Raw material mixture for the manufacture of large-sized billets of superhard composite material. Patent RF 2750448. 2021-06-28.

\section{INFORMATION ABOUTTHE AUTHORS}

Leonid A. Ivanov - Cand. Sci. (Eng.), Vice President of the Russian Academy of Engineering, Member of the International Journalist Federation; Moscow, Russia, L.a.ivanov@mail.ru, https://orcid.org/0000-0001-9513-8712

Li D. Xu - Ph.D., Prof., Old Dominion University, of Information Technologies \& Decision Sciences Department; Institute of Electrical and Electronics Engineers (IEEE), Norfolk, Virginia, USA, LXu@odu.edu, https://orcid.org/0000-0002-3263-5217

Konstantin E. Razumeev - Dr. Sci. (Eng.), Prof., Advisor to the Rector's Office of the A.N. Kosygin Russian State University (Technology. Design. Art), Moscow, Russia, ker2210@yandex.ru, https://orcid.org/0000-0003-4308-3363

Valentina M. Feoktistova - Cand. Sci. (Econ.), Assistant Professor, Educational Computer Center, Russian State University of Tourism and Service, Cherkizovo, Moscow region, Russia, vfeoktistova@gmail.com, https://orcid.org/0000-0001-8880-8941

Petr S. Prokopiev - Financial University Under the Government of the Russian Federation, PhD Student at the International Economic Relations Faculty, Moscow, Russia, prokopiev2012@gmail.com, https://orcid.org/0000-0001-6305-4135

\section{CONTRIBUTION OF THE AUTHORS}

The authors contributed equally to this article.

\section{The authors declare no conflicts of interests.}

The article was submitted 02.09.2021; approved after reviewing 24.09.2021; accepted for publication 27.09.2021. 